\title{
Development of Home-Based Exercise Mobile Application for Patients with Peripheral Artery Disease
}

\author{
Yesol KIM ${ }^{\mathrm{a}}$, Mihui KIM ${ }^{\mathrm{a}}$, Gi Wook RYU ${ }^{\mathrm{a}}$ and Mona $\mathrm{CHOI}^{\mathrm{b}, 1}$ \\ ${ }^{a}$ College of Nursing and Brain Korea 21 FOUR Project, \\ Yonsei University, Seoul, Korea \\ ${ }^{\mathrm{b}}$ College of Nursing and Mo-Im Kim Nursing Research Institute, \\ Yonsei University, Seoul, Korea
}

\begin{abstract}
This study aimed to develop a home-based exercise application to improve the exercising performance in patients with peripheral artery disease. This application is based on a theoretical framework and is paired with a wearable device, Fitbit Charge4. The application comprised five main categories with 30 screens: login, a record of exercise, goal achievement, information, and motivation. Using the application to nursing practice can manage and encourage patients to exercise without limitations of time and place.
\end{abstract}

Keywords. Exercise, mobile applications, peripheral arterial disease, telemedicine, wearable electronic devices

\section{Introduction}

Peripheral artery disease (PAD) is a chronic atherosclerotic obstructive disease with asymptomatic, intermittent claudication, and critical limb ischemia [1]. Patients with PAD experience ischemic pain during walking, which affects functional impairment and lower quality of life $[2 ; 3]$. Supervised exercise therapy (SET) performed in patients with PAD improves functional status and decreased leg symptoms [4]; however, it can be burdensome about accessibility and cost [5]. In this sense, home-based exercise therapy (HBET) may be a reasonable alternative to SET more accessible and accepted [4]. However, low adherence in HBET is challenging to achieve the effectiveness of exercise through behavioral changes [6]. Mobile health technology is an intervention strategy that can increase exercise adherence, collect behavioral health information such as daily physical activity, and provide individualized interventions regardless of time and place $[6 ; 7]$. The information-motivation-behavioral skills (IMB) model is a framework that describes the increase in health behavior by increasing health behavior skills through providing information and motivation [8]. Therefore, this study aimed to develop HBET mobile application based on the IMB model to improve the exercise performance in patients with PAD.

\footnotetext{
${ }^{1}$ Corresponding Author, Mona Choi, College of Nursing and Mo-Im Kim Nursing Research Institute, Yonsei University, Seoul, Korea.
} 


\section{Methods}

We developed a mobile application based on the IMB model [8] to monitor activities and encourage exercise in conjunction with a wearable device, Fitbit Charge 4 (Fitbit, Inc., San Francisco, CA), to change the health-related behavior of patients with PAD. In addition, this study developed a mobile application to identify, design, develop, and prototype based on the Mobile Application Development Lifecycle Model [9].

\section{Results}

\subsection{Identification}

We investigated effective exercise methods with a high level of evidence in PAD patients through literature review and the $2016 \mathrm{AHA} / \mathrm{ACC}$ guideline for PAD [5]. As a result, the volume, intensity, frequency, and duration of the exercise were determined as targets. In addition, needs assessments related to patients' exercise were investigated through interviews $(n=9)$ and surveys $(n=138)$. According to an interview among nine PAD patients, it was found that they want to use an application expected to be a companion and supporter for exercise at home and an application with functions to monitor and feedback their exercise and emotional status. Participants with PAD had a high demand for exercise-related information. Additionally, they expected mobile applications to include education about exercise and function to check their status, and they expressed willingness to use mobile applications.

\subsection{Design}

We designed the structure and functions of the mobile application. The mobile application consisted of five categories: login, a record of exercise, goal achievement, information, and motivation. For motivation, daily and weekly exercise goals for time and number of steps were set. Furthermore, a text message algorithm was designed to deliver the message via in-application push, depending on whether the daily and weekly goals were achieved.

\subsection{Development}

After our research team designed a home-based exercise mobile application for patients with PAD, an application development team developed application and dashboard systems from January to March 2021. The mobile application was developed using Ionic Framework version 5 and Cordova version 9; the operating environment is Android version 5.0 or higher. The mobile application was comprised of five main categories with 30 screens. The five main categories and each content are as follows: (1) login registration and login with ID and password; (2) a record of exercise - check the number of steps using Fitbit charge 4 and time using start/stop button in an application; (3) goal achievement - setting daily/weekly exercise goals and giving reward badges when achieving goals; (4) information - exercise and disease-related education video; and (5) motivation - sending in-application push regarding daily/weekly exercise goals according to text message algorithm. 


\subsection{Prototyping}

It was implemented that mobile application for patients with PAD in a real mobile environment such as an android phone. First, researchers tested functionality, connectivity with Fitbit charge 4, overall layout, and design of mobile applications. Then, revision and modification were performed by requesting the programmer for errors and corrections found in the mobile application.

\section{Conclusions}

The present study develops a home-based exercise mobile application based on the IMB model for patients with PAD. Using the mobile application developed based on the theoretical framework in nursing practice can manage and encourage patients to exercise without limitations of time and place or the need for additional nursing personnel. In addition, the mobile application, which provides exercise and disease-related information and motivates them through text messages, may enhance patients' positive behavior changes such as exercise performance.

\section{Acknowledgements}

This research was supported by the Basic Science Research Program through the National Research Foundation of Korea (NRF) funded by the Ministry of Education (2019R1A2C1007185) and the Brain Korea 21 FOUR Project funded by National Research Foundation (NRF) of Korea, Yonsei University College of Nursing.

\section{References}

[1] Schirmang TC, Ahn SH, Murphy TP, Dubel GJ, Soares GM. Peripheral arterial disease: Update of overview and treatment, Med Health R I 92 (2009), 398-402.

[2] Chaudru S, Jehannin P, de Müllenheim PY et al. Using wearable monitors to assess daily walking limitations induced by ischemic pain in peripheral artery disease. Scand J Med Sci Sports 29 (2019), 1813-1826.

[3] Hiatt WR, Fowkes FG, Heizer G et al. Ticagrelor versus clopidogrel in symptomatic peripheral artery disease. N Engl J Med 376 (2017), 32-40.

[4] Gerhard-Herman MD, Gornik HL, Barrett C et al. 2016 AHA/ACC guideline on the management of patients with lower extremity peripheral artery disease: A report of the American College of Cardiology/American Heart Association task force on clinical practice guidelines. Circulation 135 (2017), e726-e779.

[5] Gardner AW, Parker DE, Montgomery PS, Blevins SM. Step-monitored home exercise improves ambulation, vascular function, and inflammation in symptomatic patients with peripheral artery disease: A randomized controlled trial. J Am Heart Assoc 3 (2014), e001107.

[6] Argent R, Daly A, Caulfield B. Patient involvement with home-based exercise programs: Can connected health interventions influence adherence? JMIR Mhealth Uhealth 6 (2018), e47.

[7] Paldán K, Simanovski J, Ullrich G et al. Feasibility and clinical relevance of a mobile intervention using TrackPAD to support supervised exercise therapy in patients with peripheral arterial disease: Study protocol for a randomized controlled pilot trial. JMIR Res Protoc 8 (2019), e13651.

[8] Fisher JD, Fisher WA. The information-motivation-behavioral skills model, Emerging theories in health promotion practice and research: Strategies for improving public health 1 (2002), 40-70.

[9] Vithani T, Kumar A. Modeling the mobile application development lifecycle. In: Proceedings of the International MultiConference of Engineers and Computer Scientists, 2014, pp. 596-600. 